\title{
Spontaneous intracranial hypotension
}

\author{
S A Renowden, R Gregory, N Hyman, D Hilton-Jones
}

\begin{abstract}
The clinical features and radiological appearances of spontaneous intracranial hypotension are described in three patients and the medical literature is reviewed. Awareness of this condition and its differentiation from more sinister meningitic processes is important to avoid unnecessary invasive investigations and to allow prompt diagnosis and effective treatment.
\end{abstract}

(F Neurol Neurosurg Psychiatry 1995;59:511-515)

Keywords: spontaneous intracranial hypotension

Postural headache, as defined by the International Headache Society, is that which occurs or worsens less than 15 minutes after assuming the upright position, and disappears or improves less than 30 minutes after resuming the recumbent position. It is secondary to low intracranial pressure, which occurs most often after a lumbar puncture, craniotomy, or spinal surgery and is due to CSF leakage from a dural tear. Rarely, it may occur as a consequence of severe dehydration-for example, diabetic ketoacidosis or uraemia.

Spontaneous intracranial hypotension was first described by Schaltenbrand in $1938 .^{1}$ It typically occurs without an obvious cause but often minor trauma may be reported preceding its onset-for example, sneezing, coughing, intercourse, or a minor fall. It is important to be aware of this condition because CSF analysis and MRI abnormalities in these patients may cause unnecessary concern and result in unwarranted invasive investigations.

The three cases described illustrate certain differences in the clinical and radiological range of spontaneous intracranial hypotension and response to treatment.

\section{Case reports}

PATIENT 1

A 32 year old previously fit man developed rapid onset of severe frontal headache while standing shaving. Half an hour earlier he had received an apparently trivial blow to his forehead from his girlfriend's knee. Sexual intercourse had not taken place. The headache persisted and showed pronounced postural variation, being much worse standing up and significantly relieved, but not aborted by lying down. He also complained of an odd popping sensation in his ears.

There was no relevant medical history. Examination was normal. Unenhanced cranial CT was also normal. Lumbar puncture was difficult. There was no spontaneous flow and it was necessary to aspirate CSF. Examination of CSF showed 125 erythrocytes and 4 lymphocytes $/ \mathrm{mm}^{3}$ and the protein was raised at $1.67 \mathrm{~g} / \mathrm{l}$. Repeat lumbar puncture four days later again required CSF aspiration and examination on this occasion showed 3000 erythrocytes, 31 white blood cells $/ \mathrm{mm}^{3}$ (80\% lymphocytes), xanthochromic fluid and a raised protein concentration of $3.9 \mathrm{~g} / 1$. In retrospect it was presumed that this fluid had been aspirated from the extradural space. Routine blood tests and chest radiograph were normal. Four vessel cerebral angiography was normal. Cranial MRI showed meningeal thickening and an exudate overlying the cerebral hemispheres and small bilateral subdural effusions beneath the tentorium (fig 1). There was no mass

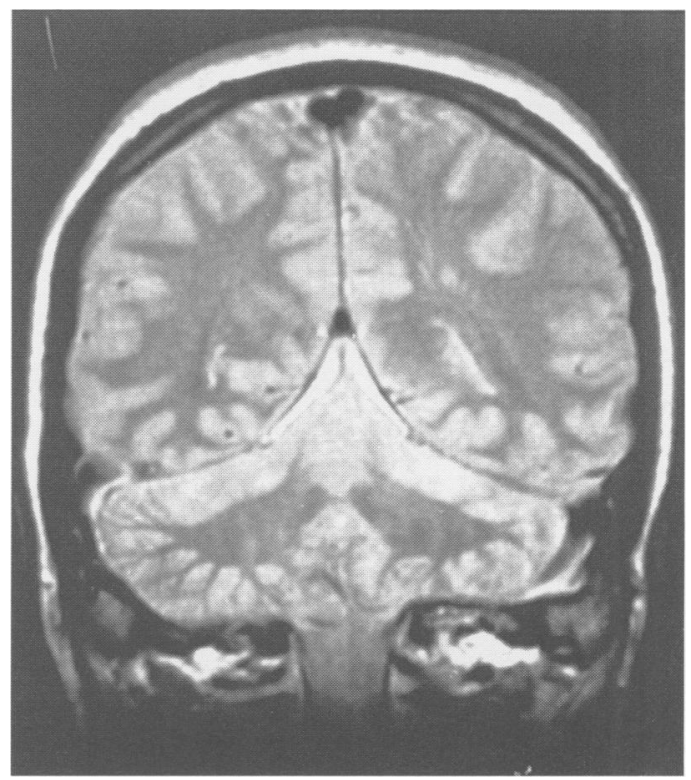

Figure 1 Coronal fast spin echo proton density cranial MRI shows high signal subdural effusions beneath the tentorium and dilatation of the cortical veins. 


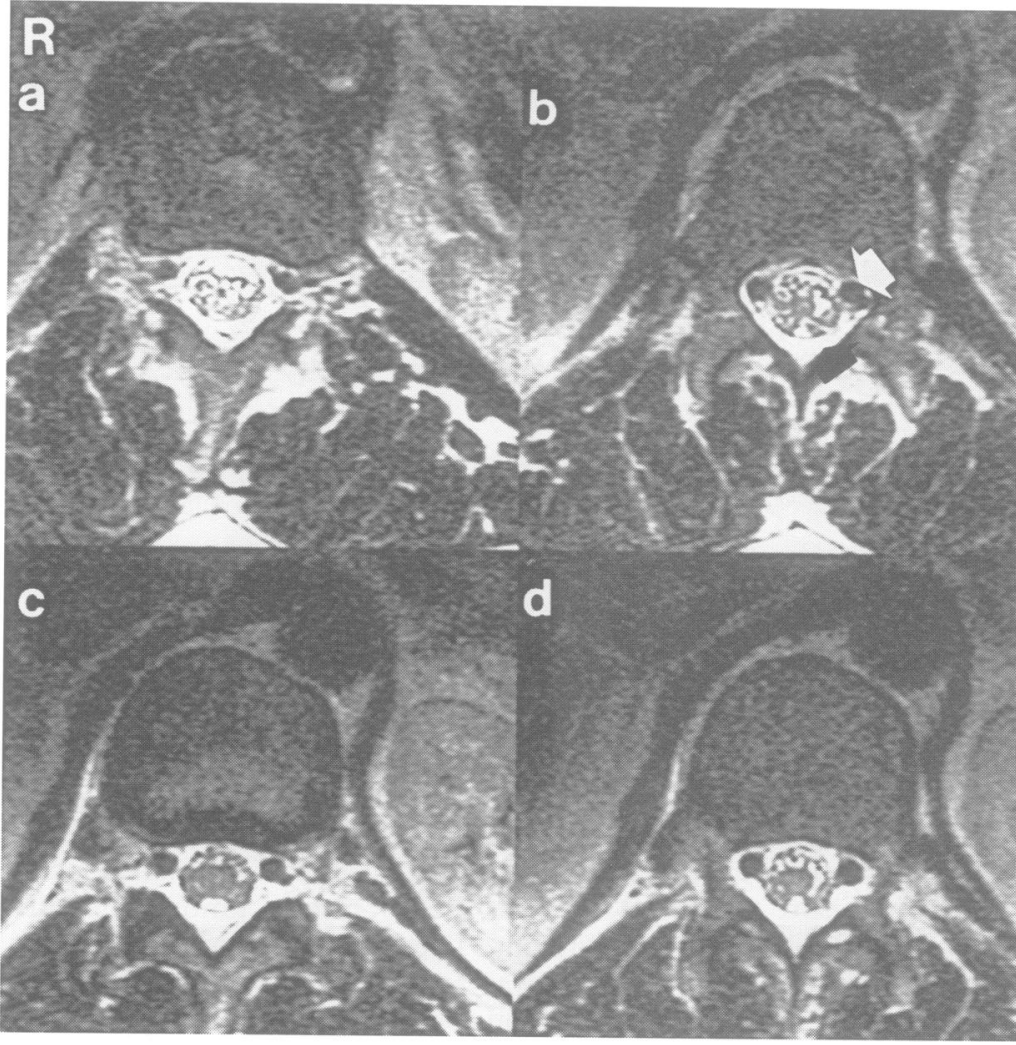

Figure 2 Fast spin echo T2W axial MRIs through the conus $(b, c$, and d) and cauda equina at L1 (a) show a collection of fluid (black arrow) outside the thecal sac in the extradural space and dilated epidural veins (white arrow). The signal returned by the extradural collection of fluid is much higher than that of epidural fat on $T 2 W$.

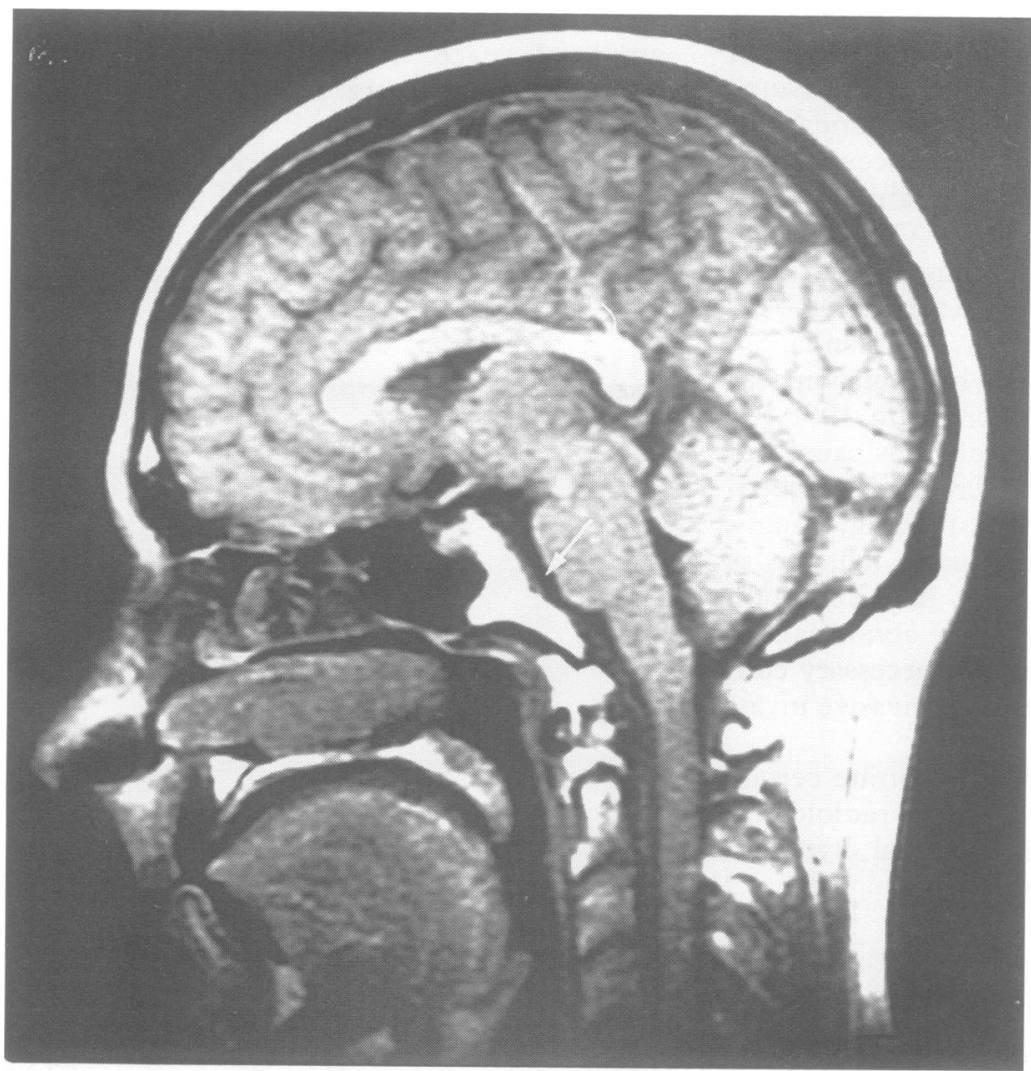

Figure $3 T 1 W$ midline sagittal cranial MRI at initial presentation, to be compared with fig 4 taken three weeks later. At presentation, the cerebellar tonsils were above the foramen magnum and note the normal position of the optic chiasm and hypothalamus relative to the pituitary fossa. Meningeal thickening and exudate are shown along the clivus (arrow). effect and no tonsillar herniation. The cortical veins were dilated. Spinal MRI showed enlarged epidural veins (fig 2) and the parasagittal images suggested that these extended from the sacrum to the mid-thoracic level. There was also a collection of fluid in the extradural space. The collection extended from the terminal sacral thecal sac to $\mathrm{C} 6 / \mathrm{C} 7$.

A diagnosis of intracranial hypotension secondary to a spontaneous spinal CSF leak was therefore made.

Myelography, performed 16 days after the onset of the headache, showed no evidence of a structural lesion or dural ectasia. The CSF contained no cells and the protein was normal at $0.6 \mathrm{~g} / \mathrm{l}$. The thecal sac was compressed by the extradural collection of CSF and traces of contrast were seen in the extradural compartment in the lumbar and thoracic regions. The headache continued unremitting. Repeat cranial MRI examination three weeks later showed the subdural effusions and cortical vein dilatation as before but now the cerebellar tonsils had descended to the foramen magnum and there was downward displacement of the optic chiasm (compare figs 3 and 4). Intravenous gadolinium showed florid meningeal enhancement over the convexities, along the tentorium, down the clivus and cervical spine (fig 4). The spinal appearances were also unchanged but now owing to the paucity of CSF within the sacral theca, when compared with the previous MRI and lumbar myelogram, the sacral nerves were clumped together erroneously simulating arachnoiditis. The headache persisted with pronounced postural dependency which rendered the patient largely bedbound and isotope cisternography was performed to localise the site of the leak before an epidural blood patch. Using $25 \mathrm{MBq}$ indium-111 labelled DTPA, isotope cisternography showed an extradural leak in the upper thoracic region (fig 5A), early appearance of isotope within the bladder indicating early absorption (fig 5B) and poor passage from the basal cisterns to the sylvian fissures and over the convexities consistent with intracranial hypotension. Twelve weeks after the onset of headache he underwent an epidural blood patch with 30 $\mathrm{ml}$ autologous blood at L1-L2 level. Within three days there was a dramatic improvement in his headache and the persistent and posturally related headache resolved after seven days. Subsequently he has experienced only brief infrequent head pains lasting only a few minutes which have shown no relation to posture.

Repeat cranial and thoracolumbar MRI two months after the blood patch showed complete resolution of the abnormalities described.

\section{PATIENT 2}

A 48 year old previously fit male patient presented with a seven day history of headache and vomiting. The headache, localised to the vertex, had come on suddenly and had increased in severity over half an hour. After 


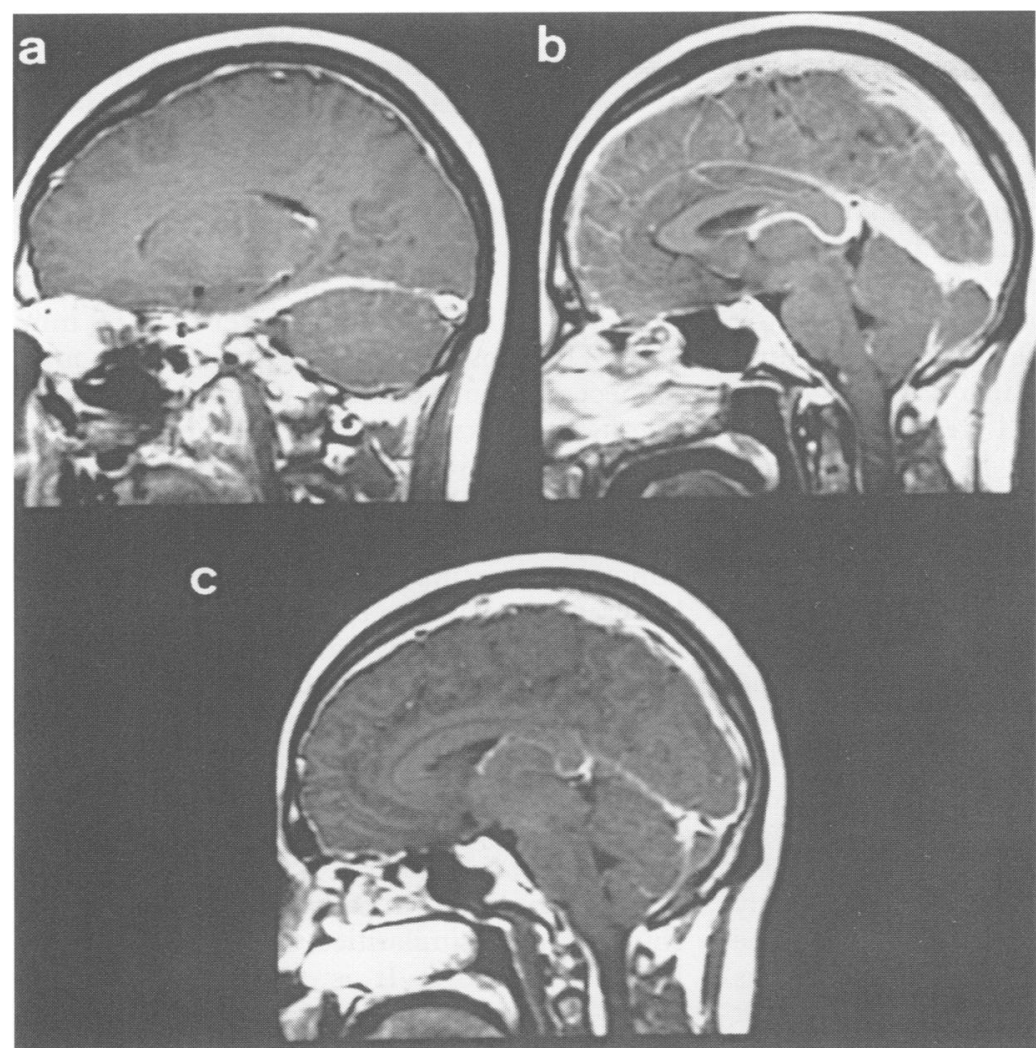

Figure 4 Parasagittal ( $a$ and c) and sagittal midline (b) gadolinium enhanced T1W $M R I s$ after three weeks show florid meningeal enhancement over the convexity, along the tentorium, clivus and down into the cervical spine. There is now relative descent of the cerebellar tonsils (compare with fig 3). Note also in (b) crowding of the optic chiasm and hypothalamus between the pituitary gland and brain.
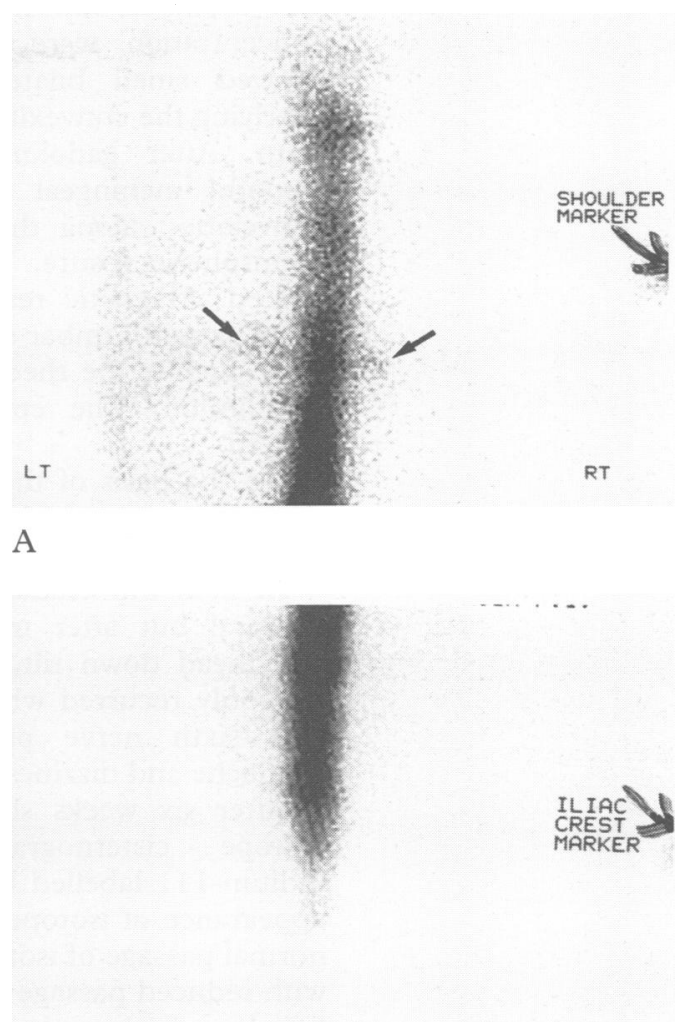

24 hours, the headache became postural. There was no photophobia or neck stiffness but he complained of tinnitus and transient hearing loss. There was no history of trauma or other precipitating event. Examination was normal. Lumbar puncture showed a CSF pressure of $60 \mathrm{~mm}$ CSF, 10 lymphocytes/ $\mathrm{mm}^{3}, 315$ erythrocytes $/ \mathrm{mm}^{3}$, and a protein concentration of $1.47 \mathrm{~g} / \mathrm{l}$. There was no xanthochromia. Routine blood tests were normal as was cranial CT.

Thoracolumbar spine MRI showed fluid outside the theca and dilated epidural veins. A diagnosis of intracranial hypotension secondary to a spontaneous spinal CSF leak was therefore made.

Cranial MRI showed an exudate over the cerebral hemispheres bilaterally, along the clivus and along the tentorium. There was no mass effect. Intravenous gadolinium was not given.

Six weeks later the headache had still not resolved and an isotope cisternogram was performed before an epidural blood patch. Cisternography, using $30 \mathrm{MBq}$ indium-111 labelled DTPA disclosed a large extradural leak at L3, the appearance of isotope in the bladder at one hour and the normal passage of isotope to the basal cisterns but reduced passage of isotope over the convexities confirming the diagnosis.

An epidural blood patch with $30 \mathrm{ml}$ of autologous blood was performed at L1/L2

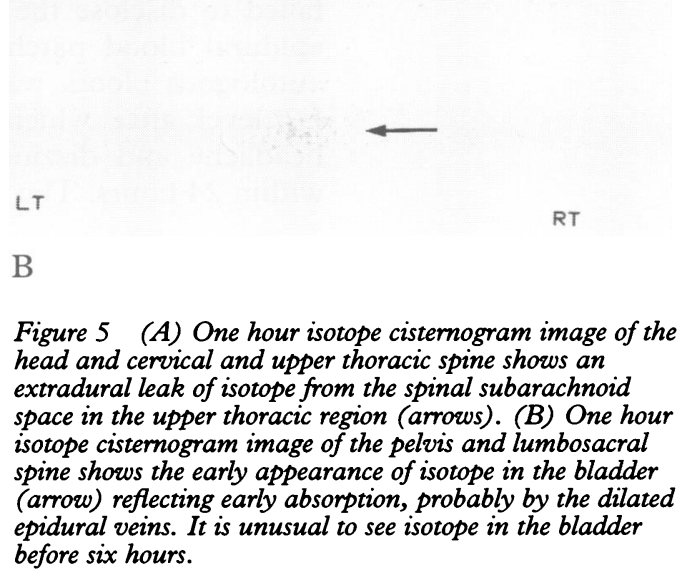

but without clinical improvement. A second blood patch using $50 \mathrm{ml}$ autologous blood at the same level was performed 24 hours later and was followed by dramatic clinical improvement and resolution of the headache. The patient remains well.

\section{PATIENT 3}

A 39 year old female patient presented with the sudden onset of a generalised headache accompanied by deafness, double vision on left lateral gaze, general clumsiness, and slight obtundation without true confusion or drowsiness. There was no history of trauma. She was a Hindu vegan who was brought up in India and moved to Britain seven years ago.

She had a partial left sixth nerve palsy, mild bilateral sensorineural hearing loss, and mild neck stiffness.

Routine blood tests and chest radiograph were normal. Lumbar puncture showed the pressure to be low at $50 \mathrm{~mm}$ CSF. The CSF 
was acellular and the glucose and protein concentration were normal. Cranial MRI showed small bilateral subdural effusions overlying the convexities and under the tentorium. After gadolinium, there was florid smooth, meningeal enhancement over the convexities, along the tentorium and interhemispheric fissure. The cortical veins were dilated. Magnetic resonance imaging of the thoracic and lumbar spine showed extradural fluid outside the theca which enhanced with gadolinium. The epidural veins were also dilated.

On the basis of the MRI findings, a diagnosis of intracranial hypotension secondary to a spontaneous spinal CSF leak was made.

At first, the headache did not seem to be postural but after treatment with bed rest with head down tilt, her headache resolved and only recurred when she stood up again. Her sixth nerve palsy resolved but the headache and dizziness on standing persisted.

After six weeks she was still not better. Isotope cisternography using $30 \mathrm{MBq}$ indium-111 labelled DTPA showed the early appearance of isotope in the bladder and the normal passage of isotope to the basal cisterns with reduced passage over the convexities but failed to disclose the site of a CSF leak. An epidural blood patch, again using $30 \mathrm{ml}$ of autologous blood, was performed at the L1L2 level after which the patient's postural headache and dizziness completely resolved within 24 hours. The patient remains well.

\section{Discussion}

The headache in spontaneous intracranial hypotension may be gradual or acute in onset and may be generalised or localised to the frontal or occipital regions. It is probably due to dilatation of the cerebral veins and meningeal vasculature as there is a reciprocal relation between CSF volume and intracranial blood volume. ${ }^{2}$ Alternatively the headache may be due to downward sagging of the brain with traction on pain sensitive structures when the patient is upright, ${ }^{3}$ or both factors may be involved. Women are affected more often than men in a ratio of 3:1 and it presents most commonly in people in their 20 s and 30 s.

Nausea, vomiting, tinnitus, vertigo, photophobia, and phonophobia are commonly associated. ${ }^{4-6}$ Ear symptoms, described by all three patients, are thought to occur because lowering CSF pressure reduces intralabyrynthine pressure and failure of transmission of high frequency sound. Additionally there may be alterations in pressure across the vestibular and cochlea aqueducts. ${ }^{78}$ Examination is often normal although bilateral sixth nerve palsies have been described, ${ }^{9}$ probably secondary to distortion of the nerve itself. Visual disturbances are reported to occur in some $23 \%$ of patients ${ }^{10}$ and the patient may complain of blurred vision and transient obscurations. Subtle defects in peripheral vision, not noticed by the patient themselves or detectable to confrontation, have also been reported. ${ }^{10}$ These are most severe in the upper nasal quadrant, but with involvement of the lower nasal and upper temporal quadrants, and may be attributed to distortion of the optic chiasm or compression or vascular congestion of the intracranial portions of the optic nerves.

Lumbar puncture is often difficult. Pressure of CSF is usually lower than $60 \mathrm{~mm}$ CSF. Dry taps are not infrequent and it may be necessary to aspirate or perform a Valsalva manoeuvre. Examination of CSF may be normal, as in patient 3 , or show mildly raised protein, increased cell count, or xanthochromia, ${ }^{461112}$ as in patients 1 and 2. The CSF abnormalities may be due to meningeal hyperaemia resulting from the low CSF pressure with diapedesis of cells into the subarachnoid space but as in patient 1 , some of the reported abnormal results may represent tapping of the extradural collections.

Meningeal biopsies in a few cases have either shown non-specific inflammation or no abnormality. ${ }^{12}$

Spontaneous intracranial hypotension shows many similarities to headache after lumbar puncture. It is thought to be the result of a spontaneous CSF leak and such leaks have often been seen on isotope cisternography. Although a spontaneous leak from an arachnoid diverticulum has been described $^{13}$ a structural lesion is seldom identified and small tears in root sleeves or Tarlov's cysts, which may occur even with minor stresses, are considered responsible. Isotope cisternography normally shows the cephalad passage of isotope to basal cisterns within two hours. If a leak is present, increasing concentration of isotope is seen at the site leak. Additionally the passage of isotope from basal cisterns to the sylvian fissure and interhemispheric cistern is reduced consistent with the low CSF pressure state. There is also rapid accumulation of isotope within the bladder indicating rapid uptake of tracer into the bloodstream, probably via the epidural venous plexus. "Brain shifts" due to low CSF pressure, such as tonsillar herniation, flattening of the pons along the clivus, and displacement of the optic chiasm and iter may be readily appreciated on cranial MRI. ${ }^{12} 14$ Subdural effusions are seen in about $10 \%$ of cases and are probably secondary to rupture of bridging veins due to the decrease in CSF volume and downward displacement of the brain. Diffuse thickening of the meninges and choroid plexus and meningeal enhancement may result from dilated leaky meningeal vessels and small vessel rupture. ${ }^{1214}$ The meningeal enhancement has been described as continuous over the convexities, along the interhemispheric fissure and tentorium but not involving the depths of the sulci. ${ }^{12}$ The meningeal enhancement and "brain shifts" may either resolve completely or improve considerably when the headache resolves. ${ }^{12}$ Awareness of this condition, its MRI appearance, and pattern of meningeal enhancement is important to avoid unnecessary investigations. Diffuse meningeal enhancement may 
be seen with malignant meningitis and infectious or granulomatous meningitis and may also be seen after craniotomy ${ }^{15}$ (after which it may persist for years) and ventricular shunting. ${ }^{16}$ It has also been reported after subarachnoid haemorrhage and venous sinus thrombosis. It occurs rarely secondary to lumbar puncture. ${ }^{17}$

Although dilatation of cortical veins has been described before, ${ }^{2}$ dilatation of the epidural veins seems not to have been previously reported. They are presumably dilated secondary to low spinal CSF pressure by a similar mechanism to that which occurs intracranially. The fluid outside the spinal thecal sac is an exudate, exhibiting contrast enhancement in patient 3 and raised protein concentration and cell content in patient 1 , and is probably the result of spinal meningeal hyperaemia.

Spontaneous intracranial hypotension is often self limiting, responding to bed rest, high fluid intake, and salt. ${ }^{12}$ The headache may, however, take up to several months to resolve and analgesics are seldom useful. In refractory cases an epidural blood patch, even if a CSF leak is not shown, as in patient 3, may be required. The blood injected into the epidural space adheres to the dural defect, forming a gelatinous seal. Autologous blood injected into lumbar epidural space ascends to the cervical region when the patient's head is lowered $30^{\circ}$ below the horizontal for 30 minutes ${ }^{18}$ and although isotope cisternography was performed routinely in each patient, this may not be necessary unless blood patch treatment has failed. ${ }^{10}$ It was necessary to repeat the blood patch in one of our patients. Placement of an epidural blood patch is generally very safe but reported complications include backache, back stiffness, and rarely, radicular discomfort and subdural haematoma. ${ }^{19-21}$ A short course of steroids, epidural or intrathecal saline infusion, or oral caffeine may also be considered. ${ }^{11}$ If all fails and a leak is found then surgical repair may be necessary.
We thank Dr Simon Blease for his input into this paper. We also thank the radiographers at the Oxford MRI unit for performing the MRI examinations and the nuclear medicine department, especially Dr Jeremy Berger and Dr Basil department, especially Dr Jeremy Berger and Dr Basi
Shepstone at the John Radcliffe hospital, for performing the Shepstone at the John
isotope cisternograms.

1 Schaltenbrand G. Neure anschauungen pathophysiologie der liquorzirkulation. Zentralblung 1938;3:290-300.

Burrows $G$. On disorders of the cerebral circulation London 1846. From Lundberg N. The saga of the Monro-Kellie Doctrine. In: Ishie S, Nagai H, Brocks $M$, eds. Intracranial pressure $V$. Berlin: Spinger-Verlag, 1983:68-75.

3 Wolff HG. Headaches and other head pain, 2nd ed. New York: Oxford University Press, 1963:96-125.

4 Bell WE, Joynt RJ, Sahs AL. Low spinal fluid pressure syndromes. Neurology 1961;10:512-21.

syndromes. Neurology 1961,10 . cerebral spinal fluid pressure. Neurology 1958;8:157-63.

Lasater GM. Primary intracranial hypotension. Headache 1970;10:63-6.

7 Hesser S. Two cases of so-called aliquorrhea. Acta Med Scand 1946; (suppl 169-170):758-68.

8 Lindqvist T, Moberg E. Spontaneous hypoliquorrhea Acta Med Scand 1949;132:556-61.

9 Berlit P, Berg-Dammer E. Abducens palsy in spontaneous intracranial hypotension. Neurology 1994;44:1552.

10 Horton JC, Fishman RA. Neurovisual findings in the syndrome of spontaneous intracranial hypotension from dural cerebrospinal fluid leak. Ophthalmology 1994;101: 244-51.

11 Rando TA, Fishman RA. Spontaneous intracrania hypotension: report of two cases and review of the hypotension: report of two cases

12 Pannullo SC, Reich JB, Krol G, Deck MDF, Posner JB. MRI changes in intracranial hypotension. Neurology 1993;43:919-26.

13 Schievink WI, Reimer R, Folger WN. Surgical treatment of spontaneous intracranial hypotension associated with a spinal arachnoid diverticulum. $\mathcal{F}$ Neurosurg 1994;80 736-9.

14 Fishman RA, Dillon WP. Dural enhancement and cerebral displacements secondary to intracranial hypotension. Neurology 1993;43:609-11.

15 Burke JW, Podrasky AE, Bradley WG. Meninges: benign postoperative enhancement on MR images. Radiology 1990;174:99-102.

16 Martin DS, Awwad EE, Pittman T. Meningeal fibrosis appearing shortly after ventricular shunting. AfNR Am f Neuroradiol 1990;11:617-8.

17 Mittl RL, Yousem DM. Frequency of unexplained meningeal enhancement in the brain after lumbar meningeal enhancement in the brain after lum
puncture. $A \mp N R A m \mathcal{F}$ Neuroradiol 1994;15:633-8.

18 Fishman RA. Intracranial hypotension. Neurology 1994, 44:1981.

19 Cornwall RD, Dolan WM. Radicular back pain following lumbar epidural blood patch. Anesthesiology 1975;43: 692-3.

20 Ostheimer GW, Palahnuik RJ, Shnider SM. Epidura blood patch for post-lumbar-puncture headache. Anesthesiology 1983;38:481-4.

21 Walpole JB. Blood patch for spinal headache. A recurrence and a complication. Anaesthesia 1975;30:783-5. 